Rev Mov Cient. 2016; 10(2): 10-20

ISSN: 2011-7191

ISSN: 2463-2236 (En línea)

\title{
USO DE LA VENTILACIÓN MECÁNICA NO INVASIVA EN DOS UNIDADES DE CUIDADO INTENSIVO DE BOGOTÁ CON RELACIÓN A REFERENTES INTERNACIONALES
}

\section{USE OF NON-INVASIVE MECHANICAL VENTILATION IN TWO INTENSIVE CARE UNITS OF BOGOTA IN RELATION TO INTERNATIONAL REFERENCES}

\author{
Yuli Marcela Aroca \\ Yury Alejandra Melo ${ }^{2}$ \\ Johana Paola Nieto Mestre ${ }^{3}$ \\ Luz Ángela Alejo de Paula ${ }^{4}$
}

Fecha de Recepción: 04 de abril de 2016

Fecha de Aceptación: 16 de diciembre de 2016

Citar como:

Aroca Y, Melo Y, Nieto-Mestre J \& Alejo-de Paula L. (2016). Uso de ventilación mecánica no invasiva en dos unidades de cuidado intensivo de Bogotá con relación a referentes internacionales. Revista Movimiento Científico 10(2): 10-20.

Aroca Y, Melo Y, Nieto-Mestre J \& Alejo-de Paula L. (2016). Uso de ventilación mecánica no invasiva en dos unidades de cuidado intensivo de Bogotá con relación a referentes internacionales. Revista Movimiento Científico [en línea] 2016, [fecha de consulta: dd//mm/ aaaa]; 10(2): 10-20. Disponible desde: http://revistas.iberoamericana.edu.co/index.php/Rmcientifico/issue/archive.

\section{RESUMEN}

Introducción: la Ventilación Mecánica No Invasiva (VMNI) entrega un soporte ventilatorio mecánico sin la utilización de una vía aérea artificial y es ampliamente utilizada al interior de las Unidades de Cuidado Intensivo. Objetivo: caracterizar el uso de la VMNI en dos UCI en la ciudad de Bogotá a la luz de los estándares internacionales que la soportan. Materiales y métodos: estudio descriptivo, observacional, con una muestra por conveniencia dirigido a población adulta al interior de la Unidad de Cuidado Intensivo (UCI), con información recopilada de 31 pacientes que cumplían con los requisitos de inclusión y estaban sometidos al uso de la VMNI en un tiempo comprendido entre octubre y diciembre de 2015. Resultados: el manejo de los pacientes sometidos a VMNI en estas dos UCI de la cuidad de Bogotá va ligado a los lineamientos internacionales referenciados en la literatura mundial. Se resalta el cumplimiento de: indicaciones, interfaces e instauración de los parámetros ventilatorios iniciales, entre ellos la titulación de la Presión Positiva al final

Fisioterapeuta. Estudiante de Posgrado. Especialización en Fisioterapia en Cuidado Crítico. Facultad de Ciencias de la Salud. Corporación Universitaria Iberoamericana.

2 Fisioterapeuta. Estudiante de Posgrado. Especialización en Fisioterapia en Cuidado Crítico. Facultad de Ciencias de la Salud. Corporación Universitaria Iberoamericana.

3 Fisioterapeuta. Estudiante de Posgrado. Especialización en Fisioterapia en Cuidado Crítico. Facultad de Ciencias de la Salud. Corporación Universitaria Iberoamericana.

4 Fisioterapeuta. Universidad Nacional de Colombia. Candidata a Magíster en Educación con énfasis en Ciencias de la Salud. Especialista en Fisioterapia en Cuidado Crítico. Docente Programa de Fisioterapia. Facultad de Ciencias de la Salud. Corporación Universitaria Iberoamericana. Correo electrónico: luz.alejo@iberoamericana.edu.co 
de la Espiración (PEEP) de 8-12 $\mathrm{cmH}_{2} \mathrm{O}$ para mejorar el estado de oxigenación y mantener capacidad residual funcional, la presión de soporte establecida entre 10 a $12 \mathrm{cmH}_{2} \mathrm{O}$ para disminuir trabajo respiratorio por parte de los músculos respiratorios, y el nivel de sensibilidad fijado en -2L/s, con el fin de disminuir y evitar los episodios de asincrónica paciente ventilador, entre otros. Conclusiones: se deben realizar más estudios en el manejo de la VMNI en Colombia, que permitan a profesionales e instituciones evaluar y comparar sus protocolos de manejo y niveles de adherencia en relación a la literatura tanto nacional como internacional.

Palabras clave: Ventilación, Ventilación con CPAP, ventilación no invasiva, Unidades de cuidados intensivos.

\section{ABSTRACT}

Background: Non-Invasive Mechanical Ventilation (VMNI) provides mechanical ventilatory support without the use of an artificial airway and is widely used within Intensive Care Units. Objective: To characterize the use of NIMV in two ICUs in the city of Bogotá in the light of the international standards that support it. Materials and methods: A descriptive, observational study with a convenience sample aimed at the adult population inside the Intensive Care Unit (ICU), with information collected from 31 patients who met the inclusion requirements and who were submitted to the use of VMNI in a Between October and December 2015.Results: The management of patients undergoing VMNI in these two ICUs in the city of Bogotá is linked to the international guidelines referenced in the world literature. The compliance of: indications, interfaces and establishment of the initial ventilatory parameters, among them the titration of the Positive Pressure at the end of the Expiration (PEEP) of 8-12cmH2O to improve the state of oxygenation and to maintain functional residual capacity, support pressure set between 10 to $12 \mathrm{cmH} 2 \mathrm{O}$ to decrease respiratory work by the respiratory muscles, and the level of sensitivity set at $-2 \mathrm{~L} / \mathrm{s}$, in order to decrease and avoid episodes of asynchronous ventilator patient, among others. Conclusions: More studies should be carried out in the management of VMNI in Colombia, allowing professionals and institutions to evaluate and compare their management protocols and adherence levels in relation to national and international literature.

Keywords: Mechanic ventilation, Noninvasive Ventilation, Intensive Care Units.

\section{INTRODUCGIÓN}

En la actualidad la Ventilación Mecánica No Invasiva (VMNI) se define como una conducta terapéutica capaz de entregar un soporte ventilatorio mecánico sin la utilización de una vía aérea artificial; es decir, permite el uso de la vía aérea natural del paciente. Esta estrategia ampliamente usada al interior de las Unidades de Cuidado Intensivo (UCIs) en el mundo, proporciona principalmente dos modos ventilatorios básicos: la ventilación con dos niveles de presión (BiPAP) y la presión positiva Continua en la vía aérea (CPAP) (Artacho, Guzmán, López, S., García, Caballero, López, M. \& Del Campo, 2015).

Desde finales del siglo XX e inicios del siglo XXI, se ha demostrado que la utilización de la VMNI en falla respiratoria aguda tiene un alto nivel de evidencia científica, y su uso se ha extendido ampliamente a situaciones severas como la exacerbación en el paciente con Enfermedad Pulmonar Obstructiva Crónica (EPOC). Sin embargo, se ha demostrado a nivel mundial que muchas unidades de cuidados intensivos limitan su uso debido a la falta de conocimiento del equipo médico en cuanto a su adecuado manejo (Truwit \& Epstein, 2011).

Históricamente hacia los años 20 del siglo XX, el primer pulmón de acero o ventilador generador de presión negativa fue diseñado, utilizado y modificado en varias ocasiones para finalmente generalizar su uso hacia finales de 1928. En la década de los años 30 se desarrollaron sistemas como el Pneumobelt los cuales ejercían presión intermitente sobre el tórax de los pacientes, y aunque su utilización creció exponencialmente en 1952 con la epidemia del polio, también demostró la alta tasa de 
neumonitis química por microaspiraciones que estos sistemas producían.

Dado este significativo efecto deletéreo para la salud de los pacientes en cuidado crítico, a partir de esta época el uso de este tipo de ventiladores según Uña y colaboradores (2005) comenzó a decaer y a volverse casi obsoleto. A la par, los ventiladores mecánicos pero con aplicación de presión positiva invasiva fueron utilizados por primera vez en los años 30 cuando se utilizó para manejar el edema agudo de Pulmón con resultados positivos. A finales de la década, puntualmente en 1938 fue creado el primer ventilador mecánico que aportaba presión positiva, pero de forma no invasiva, dicho artefacto fue muy criticado dado que su uso era manual y complejo; por tanto, sólo hacia los años 60 se empezó a manejar la VMNI en pleno, inicialmente en pacientes neuromusculares usando máscaras nasales u oro nasales simples y a mediados de los años 80 e inicios de los 90 cuando las máscaras nasales intentaron garantizar una adecuada entrega de flujo al paciente, dado confort y adherencia, se inició la época de gran despliegue sobre el uso de la VMNI (Truwit et al, 2011).

Hoy en día la VMNI se considera una de las principales estrategias para reducir la tasa de intubación y las tasas de mortalidad en pacientes con EPOC exacerbado y edema pulmonar cardiogénico, pero su uso e impacto en pacientes con falla respiratoria aguda hipoxémica no hipercápnica, en ausencia de enfermedad pulmonar o cardiaca de base está en construcción.

Estudios controlados aleatorizados demuestran el incremento creciente en el uso de la VMNI en pacientes en falla respiratoria aguda hipoxémica como primera línea de soporte en al menos el $30 \%$ de los pacientes que la presentan, señalando que esta aumenta la sobrevida de los pacientes al evitar la intubación orotraqueal, pero de igual manera puede llegar a ser una mala elección en el momento de retrasar una intubación orotraqueal necesaria (Thille, Contou, Fragnoli, Córdoba-Izquierdo, Boissier \& Brun-Buisson, 2013) (Ferrer, Esquinas, León, González \& Alarcón, 2003).

De igual manera Antonelli y colaboradores en 2007 demostraron que su uso temprano en pacientes que presentan Síndrome de Distress Respiratorio Agudo (SDRA), mejora los índices de oxigenación y éxito en más del $50 \%$ de los pacientes en los que ha sido utilizada de manera temprana. Otras investigaciones han enseñado resultados poco favorables de uso en pacientes con índices de oxigenación (PaFI) menor a 100 , donde se vislumbra que dicha población tiene un $84 \%$ de probabilidad de fallar con esta herramienta. También se ha evidenciado un aumento en su utilización en pacientes Inmunosuprimidos o en Post-operatorio de cirugía que presentan falla respiratoria aguda hipoxémica, pero es importante tener en cuenta que se ha demostrado que los pacientes con falla Hipoxémica son más propensos a fallar durante la VMNI (Demoule, Girou, Richard, Taillé, \& Brochard, 2006).

Delclaux y colaboradores en un estudio desarrollado en 2000, probaron que los pacientes con falla hipoxémica a quienes se les maneja con VMNI presentan de un 25 a 36\% más de probabilidad de intubación que la media promedio de los pacientes que habitualmente usan esta estrategia ventilatoria. Esto no significa que aumenta directamente la mortalidad, se traduce en aumento del número de eventos adversos relacionados con su uso, por lo cual el uso amplio y generalizado en todo tipo de pacientes se ve limitado y debe ser estudiado a fondo para garantizar los mejores desenlaces (Keenan, Sinuff, Burns, Muscedere, Kutsogiannis \& Mehta, 2011).

Hannan y colaboradores en 2014 publicaron una revisión sistemática donde analizaron los efectos de la VMNI en miras de la calidad de vida de los pacientes sometidos a esta terapéutica, encontraron que los mismos pacientes referían que con el uso de la VMNI se mejoraba su disnea, la calidad del sueño, la función física, la salud mental y emocional y la función social.

Todo lo anterior reta diariamente a los profesionales de la salud y en especial a los fisioterapeutas especialistas en cuidado crítico, en cuanto al manejo de esta estrategia terapéutica, puesto que en el momento de su implementación el ¿̇cuándo?, ¿cómo? y ċquienes? son preguntas cruciales y desencadenantes de un gran éxito o rotundo fracaso frente a su uso en las UCIs. Por tanto este estudio busca, por una parte, 
revisar los lineamientos o recomendaciones aceptadas internacionalmente en cuanto al uso de la VMNI, y por otro lado, describir su uso actual a luz de dichos referentes, al exponer su implementación en dos UCIs de la cuidad Bogotá.

Finalmente esta investigación al realizar una comparación básica de dicha información, vislumbrara si el manejo de la VMNI en las dos UCIs de la cuidad de Bogotá, están o no, en concordancia con las guías o referentes internacionales. Esta información será relevante en el gremio de la medicina cítrica y el cuidado intensivo con relación a la implementación, adherencia y evaluación periódica de los protocolos de atención se utilizan, garantizando a la población que habitualmente requiere la internación en una UCI, unas intervenciones terapéuticas con la menor comorbilidad.

\section{MATERIALES Y MÉTODOS}

Se realizó un estudio descriptivo transversal de corte observacional, con un muestreo no probabilístico por conveniencia donde se seleccionaron dos UCIs en la ciudad de Bogotá y se caracterizó el uso de la VMNI con relación a estándares internacionales.

La población de este estudio estuvo conformada por 31 pacientes que cumplieron con los criterios de inclusión, dentro de los cuales se contempló que fueran pacientes Mayores de 18 años, sometidos a VMNI entre el 1 de octubre de 2015 y el 18 de diciembre de 2015, que no fueran pacientes que manejaran VMNI domiciliaria o como Tratamiento Crónico.

Durante la investigación se utilizó un formato de recolección de datos el cual fue diseñado y aplicado por las autoras del estudio. Las variables de análisis se agruparon en tres categorías: demográfica, de valoración inicial y de intervención.

En la variable demográfica, se contempló la edad, el género y diagnóstico médico de ingreso; en la variable de valoración de inicio de la VMNI, se examinó los gases arteriales y finalmente en la variable de intervención se desglosó el uso de la VMNI, teniendo en cuenta el modo ventilatorio y los parámetros ventilatorios utilizados. Para su análisis estadístico y presentación descriptiva se utilizó la hoja de datos de Excel.

\section{RESULTADOS}

El 68\%(n=21) de la población era de género masculino en relación al $32 \%(n=10)$ de género femenino; en rangos de edad desde 18 hasta 86 años de edad con una edad promedio de 47.2 años, el 39\%(n=12) se encuentra dentro del rango de edad de 18 a 35 años, el $10 \%(n=3)$ en el rango de edad de 36 a 50 años, el $23 \%(\mathrm{n}=7)$ hace parte del rango de edad de 51 a 65 años y el restante $29 \%(n=9)$, representa el rango de edad de más de 66 años.

Como diagnósticos de ingreso descritos en la Tabla 1. se puede clasificar a su vez las patologías como enfermedades intrapulmonares y extrapulmonares se encontró que un $61 \%(\mathrm{n}=19)$ correspondía a patologías de origen intrapulmonares, y el 39\% $(n=12)$ a patologías extrapulmonares.

Tabla 1. Diagnósticos de Ingreso

\begin{tabular}{lc}
\hline \multicolumn{1}{c}{ Diagnóstico } & Pacientes \\
\hline Edema pulmonar cardiogénico isquémico & 1 \\
\hline EPOC Exacerbado & 6 \\
\hline Falla Respiratoria POP & 1 \\
\hline Edema pulmonar cardiogénico de origen valvular & 3 \\
\hline Depresión respiratoria & 1 \\
\hline Neumonía Bacteriana & 4 \\
\hline Neumonía Viral & 2 \\
\hline SAHOS & 3 \\
\hline Polineuropatías & 2 \\
\hline POP de Apendicectomía & 5 \\
\hline Traumatismo Intracraneal & 2 \\
\hline Tumor Maligno de Esófago & 1 \\
\hline
\end{tabular}

Fuente: elaboración propia (2015).

Las patologías que afectan la respiración de forma directa o indirecta se pueden clasifican en disfunciones hipoxémicas e hipercápnicas, la afectación hipoxémica está dada por la imposibilidad del parénquima pulmonar de realizar un intercambio gaseoso efectivo, esto se da 
en patologías como la neumonía, el edema pulmonar cardiogénico y el SDRA entre otras, en este estudio se encontró que el 39\%(n=12) de los pacientes usaron VMNI por presentar falla respiratoria hipoxémica. En cuanto a la afectación respiratoria hipercápnica la cual se presenta cuando hay una disfunción o falla en la bomba respiratoria en patologías como EPOC, estados asmáticos, insuficiencias respiratoria postoperatorios entre otros, en los pacientes del estudio el $35 \%(n=11)$ este tipo de afección al momento de iniciar la VMNI Con el objetivo principal de soportar la carga extra de los músculos respiratorios para así mejorar la ventilación alveolar que permita la disminución de los niveles de CO2 (Keenan et al., 2011). El 26\% $(n=8)$ restante de los pacientes la indicación como protocolo de extubación. Todo lo anterior se puede revisar en la Gráfica 1.

Gráfica 1. Clasificación de las patologías

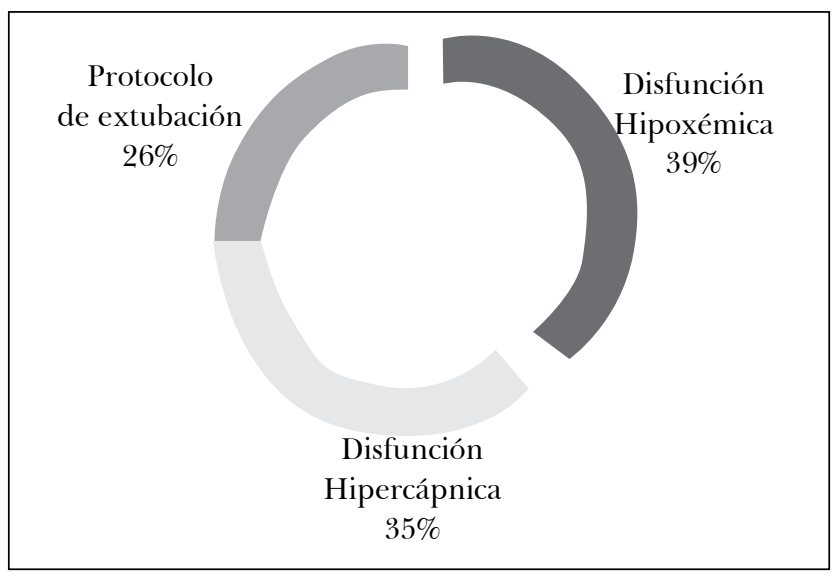

Fuente: elaboración propia (2015).

Se establecieron tres posibles escenarios donde la VMNI estaría indicada, esto se describe en la Tabla 2. Las dos primeras indicaciones basadas en pruebas de gases arteriales tomadas a los pacientes. En los gases arteriales reportados durante el momento de la VMNI se evidencia que el $84 \%(n=26)$ de los pacientes presentaban una acidosis respiratoria, y el $16 \%$ restante además de una acidosis respiratoria presentaban compromiso leve de la oxigenación, aun cuando ya se les había iniciado la terapia con VMNI.
Tabla 2. Indicaciones de uso de la VMNI

\begin{tabular}{ll}
\hline \multicolumn{2}{c}{ Indicación } \\
\hline Falla Respiratoria Hipoxémica & $39 \%(\mathrm{n}=12)$ \\
\hline Falla Respiratoria Hipercápnica & $35 \%(\mathrm{n}=11)$ \\
\hline Protocolo de Extubación & $26 \%(\mathrm{n}=8)$ \\
\hline
\end{tabular}

Fuente: elaboración propia (2015).

Con relación al tiempo de uso de la VMNI, se encontró que la totalidad de los pacientes utilizaron la VMNI por un tiempo mayor de 24 horas, logrando en ocasiones completar hasta su uso por 7 días de forma continua con lapsos de descanso para su alimentación. En la Tabla 3. se encuentran descritos los días que los pacientes estuvieron sometidos a VMNI.

Tabla 3. Tiempo de uso de la VMNI

\begin{tabular}{|c|c|}
\hline & Pacientes \\
\hline Días de uso de la VMNI & $\%$ \\
\hline 1 & $32 \%(\mathrm{n}=10)$ \\
\hline 2 & $16 \%(\mathrm{n}=5)$ \\
\hline 3 & $29 \%(n=9)$ \\
\hline Más de 4 días & $24 \%(\mathrm{n}=7)$ \\
\hline
\end{tabular}

Fuente: elaboración propia (2015).

En cuanto al uso de la interfaz, como se observa en la Gráfica 2, se encontró que el 94\% $(n=29)$ de los pacientes fueron manejados con interfaces Oro nasales y el $6 \%(n=2)$ con interfaces nasales.

Al revisar los parámetros ventilatorios utilizados, se evidenció que una de las modalidades más utilizadas en las UCIs es la modalidad de presión positiva continua en la vía aérea (CPAP), utilizada en el $32 \%(n=10)$ de los pacientes; en la Tabla 4 se encuentra la descripción de cada una de las modalidades ventilatorias utilizadas en dichas unidades. 
Gráfica 2. Tipo de interfaz utilizada

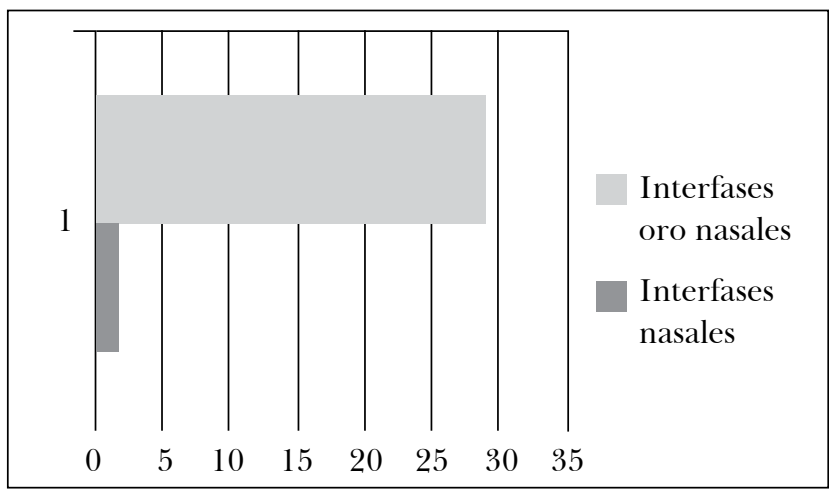

Fuente: elaboración propia (2015).

Para la mejor valoración de los parámetros ventilatorios utilizados, se dividió a los pacientes en aquellos que presentaban patologías intrapulmonares obstructivas, patologías intrapulmonares restrictivas y patologías extra-pulmonares.

Tabla 4. Modos ventilatorios usado en la VMNI

\begin{tabular}{lc}
\hline \multicolumn{1}{c}{ Modalidad Ventilatoria } & $\mathbf{N}^{\circ}$ de Pacientes \\
\cline { 2 - 2 } & $\%$ \\
\hline $\mathrm{CPAP} *$ & $32 \%(\mathrm{n}=10)$ \\
\hline $\mathrm{CPAP}+\mathrm{PRESIÓN} \mathrm{DE} \mathrm{SOPORTE}$ & $23 \%(\mathrm{n}=7)$ \\
\hline $\mathrm{BIPAP}+\mathrm{ST}^{* *}$ & $7 \%(\mathrm{n}=2)$ \\
\hline $\mathrm{ST}+\mathrm{AVAPS}^{* * *}$ & $6 \%(\mathrm{n}=2)$ \\
\hline
\end{tabular}

* CPAP: Presión positiva continua en la vía aérea.

**BIPAP+ST: Presión positiva en la vía aérea de doble nivel + (respiraciones espontánea / cronometrada).

***ST+AVAPS: Presión de soporte con volumen corriente garantizado + (respiraciones espontánea / cronometrada).

Fuente: elaboración propia (2015).

Los parámetros ventilatorios utilizados en las patologías obstructivas, demuestran con relación a la frecuencia respiratoria espontánea/controlada un valor promedio de 15 respiraciones por minuto (rpm), con un valor mínimo de 10rpm y un máximo de 20rpm; la presión soporte programada más utilizada fue de $10 \mathrm{cmH} 2 \mathrm{O}$, con una mínima de $8 \mathrm{cmH} 2 \mathrm{O}$ alcanzando valores de hasta 12cmH2O; una presión positiva al final de la espiración (PEEP) de 8cmH2O con una máxima de
10cmH2O y mínima de 6cmH2O; y una sensibilidad de $-2 \mathrm{~L} / \mathrm{s}$.

En las patologías restrictivas la frecuencia respiratoria espontánea/controlada promedio que permitía a los pacientes manejar fue de $16 \mathrm{rpm}$, con un valor mínimo de 10rpm a un máximo de 26rpm; la presión soporte programada más utilizada fue de $12 \mathrm{cmH} 2 \mathrm{O}$, con una mínima de $8 \mathrm{cmH} 2 \mathrm{O}$; con una PEEP promedio de $10 \mathrm{cmH} 2 \mathrm{O}$ utilizando valores mínimos de $8 \mathrm{cmH} 2 \mathrm{O}$; con una sensibilidad de $-2 \mathrm{~L} / \mathrm{s}$.

En las patologías extra-pulmonares que llevaron al paciente a utilizar VMNI, con un pulmón sano, tipo de compromiso intracraneal, pop de cirugías abdominales, polineuropatías entre otras, se evidenció la utilización de una frecuencia respiratoria espontánea/controlada promedio que se le permitía a los pacientes de 16rpm, con un valor mínimo de $11 \mathrm{rpm}$ a un máximo de 20rpm; la presión soporte programada más utilizada fue de $10 \mathrm{cmH} 2 \mathrm{O}$, con una máxima de $12 \mathrm{cmH} 2 \mathrm{O}$; con una PEEP promedio de $8 \mathrm{cmH} 2 \mathrm{O}$ utilizando valores mínimos de hasta $6 \mathrm{cmH} 2 \mathrm{O}$; con una sensibilidad de -2L/s.

\section{DISCUSIÓN}

En la actualidad la VMNI es uno de los tratamientos de primera línea en la atención de los pacientes con insuficiencia respiratoria aguda en las UCIs, en este tipo de pacientes el objetivo principal, con esta intervención, es evitar la intubación orotraqueal y las complicaciones derivadas de ésta, disminuir el trabajo respiratorio impuesto a los músculos de la respiración por la falla, corregir los niveles de hipoxemia y la consecuente acidosis entre otros (Masclans, Pérez, Almirall, Lorente, Marqués \& Socias, 2013).

En las unidades de estudio analizadas para esta investigación se observó que el $68 \%(\mathrm{n}=21)$ de la población era de género masculino, esto puede ser explicado por factores ambientales, psicológicos y físicos ligados al género, estudios en el área han reconocido la prevalencia en el ingreso a las UCIs del género masculino correlacionado directamente con el tabaquismo como unos de los factores predisponentes en el daño del tejido y función pulmonar en 
los hombres (Rodríguez, Muguercia, Fabré, Esquivel \& Zamora, 2012).

Al momento de instaurar la VMNI se tienen en cuenta el tipo de patología respiratoria a tratar, bien sea patologías que cursan con procesos hipoxémicos o hipercápnicos, teniendo claro que las patologías que cursan la afectación de la ventilación alveolar son las de mayor beneficio y aprovechamiento terapéutico. $\mathrm{Al}$ revisar los lineamientos internacionales las afectaciones hipercápnicas tienen un nivel de recomendación 1A (Keenan et al, 2011) para el uso de la VMNI.

Por el contrario las afectaciones hipoxémicas puras tienen variedad en sus recomendaciones de uso, prevaleciendo calificaciones medias y medias bajas, con niveles de recomendaciones que oscilan entre 2B y NA (Keenan et al, 2011). Si se revisan los datos estadísticos recopilados en este estudio se puede aseverar que su uso cumple con estos lineamientos, dado que el $84 \%(n=26)$ de los pacientes presentaban afectaciones hipercápnicos y el $16 \%$ restante presentaban compromiso de la oxigenación sumando en la mayoría de los casos con hipoventilación alveolar (Keenan et al, 2011).

$\mathrm{Al}$ relacionar individualmente las patologías que llevaron al uso de la VMNI en este estudio se destaca que las enfermedades pulmonares con procesos obstructivos con presencia de insuficiencia respiratoria hipercápnica, fueron las patologías de mayor uso, representado en un 29\%, siendo estas patologías puntualmente donde los referentes internacionales generan una recomendación de uso 1A. (Keenan et al, 2011), dado que disminuye la tasa de intubación orotraqueal y a su vez la estancia en UCI (Parola, Romani, Petroianni, Locorriere, \& Terzano, 2012). Es llamativo que pese a los referentes internacionales (nivel 2C y NA) (Keenan et al, 2011) para el uso de la VMNI en el tratamiento de la falla respiratoria aguda posterior a la extubación, esta modalidad se usó en un $26 \%$ de los casos.

En pacientes con crisis asmáticas el uso de la VMNI no ha sido tan sistematizado debido a que en diversos metanálisis se ha expresado que en casos graves de asma que responden al tratamiento convencional es mejor no retrasar la intubación orotraqueal. Los referentes internacionales no recomiendan su uso y dentro de este estudio no se contó con ningún paciente con uso de VMNI asociado a esta clase de patología.

En neumonía y pacientes con SDRA la utilización de la VMNI no está muy apoyada y siempre se aconseja no retrasar la intubación orotraqueal debido al alto riesgo de fracaso de la VMNI (Keenan, Sinuff, Cook, \& Hill, 2004); la recomendación según los referentes internacionales con relación a la implementación de la VMNI en esta clase de patologías es casi nula, con calificadores de 1C y NA (Keenan et al, 2011), en este estudio es interesante resaltar que no se usó la VMNI en el SDRA, pero si se aplicó en 6 casos en presencia de algún tipo de neumonía.

Para pacientes con inmunosupresión se aconseja el uso de la VMNI en fases iniciales de la enfermedad con el objetivo de disminuir riesgo infeccioso de las intubaciones (Curtis, Cook, Sinuff, White, Hill \& Keenan, 2007). En los referentes internacionales se observa una recomendación $2 \mathrm{~B}$, se denota que en este estudio no se contó con pacientes con este tipo de trastorno.

La literatura mundial refiere que los modos ventilatorios de mayor uso al instaurar la VMNI son el BIPAP y CPAP. Al debatir cuál de las dos modalidades tiene un mejor desempeño en el paciente crítico la literatura mundial y los lineamientos internacionales sugieren que es fundamental tener en cuenta el objetivo de su utilización y el tipo de falla o disfunción pulmonar que llevó a su aplicación; es decir, si la causa que llevó al uso de la VMNI fue una falla o disfunción hipercápnica, el objetivo principal será mejorar la ventilación alveolar, por tanto el modo de recomendación nivel I o alta es el BIPAP; por el contrario, si la causa que llevó al uso de la VMNI fue una falla o disfunción hipoxémica, el objetivo principal será mejorar la oxigenación, por tanto el modo de recomendación nivel I o alta es el CPAP.

El modo BIPAP aplica una presión en la vía aérea en dos niveles, una en la fase inspiratoria llamada IPAP y la otra en la fase espiratoria denominada EPAP, donde 
la diferencia entre estas dos es el valor de presión de soporte que se le está entregando al paciente; en su implementación se le pueden adicionar un coadyuvantes denominado ST, este adicional genera que el ventilador inicia directamente la respiración si no detecta el esfuerzo respiratorio del paciente (Del Castillo, Galán, Gordillo \& Mateos, s/f). El EPAP tiende a estabilizar la parte superior de las vías respiratorias en el final de la espiración de modo que la vía respiratoria es suficientemente para permitir la entrega de IPAP generando un volumen o flujo inspiratorio de bajo nivel durante el esfuerzo posterior. El nivel de IPAP se establece para evitar el cierre de la vía aérea superior y la obstrucción parcial (hipopnea) durante la fase inspiratoria de la respiración.

La modalidad CPAP groso modo es la aplicación de una Presión positiva continua en la vía aérea que genera flujos entre 40-120 L/min, para compensar la demanda inspiratoria del paciente, en este modo el paciente respira espontáneamente con niveles de presión superior a la atmosférica, el volumen corriente (VT), la frecuencia respiratoria y la ventilación alveolar, cambian en el tiempo dependiendo del patrón respiratorio del paciente.

El CPAP genera un gradiente de presión positiva dentro del ciclo inspiración-espiración, esto aumenta la presión media de la vía aérea, acentúa la capacidad residual funcional (CRF) y disminuye el esfuerzo respiratorio. Desde un punto de vista hemodinámico ocurre un desplome del retorno venoso, esto justifica su uso en los casos de edema pulmonar agudo, produciendo mejoría en la disnea e intercambio gaseoso siempre y cuando no se encuentre hipercapnia ya que esta modalidad no aumenta la ventilación (Ferrero, 2008).

Al revisar lo encontrado en esta investigación, se corrobora con la literatura mundial la mayor empleabilidad de estos dos tipos de modalidad ventilatoria, el modo CPAP fue el de mayor uso representado en un 58\%, un 23\% CPAP + PS y BIPAP en solo un $7 \%$. Si se compara estos usos con los estándares internacionales ellos no hacen diferenciación de la modalidad como variable individual de uso, sino que la correlacionan con otros factores o variables.
En cuanto a los parámetros ventilatorios puntuales en el uso en la VMNI los referentes internacionales no tienen un estándar o dato para ser recomendado, por tanto estos datos se contrastaron con los reportados en la literatura mundial. Dichas referencias sugieren el inicio de la VMNI con niveles de PEEP de $6 \mathrm{cmH}_{2} \mathrm{O}$ hasta un máximo $12 \mathrm{cmH} 2 \mathrm{O}$ para mantener y optimizar la oxigenación, aunque recomienda vigilar su repercusión hemodinámica. En esta población se utilizaron niveles de PEEP con un rango un poco más altos que los referenciados anteriormente (entre 8 cmH2O y 12cmH2O) el uso de niveles de PEEP por debajo de 8 fue en muy pocos casos.

Otro parámetro interesante de analizar es la presión de soporte, definida como la tasa variable de flujo de gas capaz de generar un aumento brusco en la presión de la vía aérea del paciente, se usa para disminuir la postcarga (trabajo respiratorio) de los músculos respiratorios y así reducir las demandas energéticas de los pacientes con distensibilidades comprometidas y resistencias aumentadas (Kacmarek, 1988). La literatura mundial reporta que el nivel de presión de soporte puede ajustarse lo suficientemente alto como para que el trabajo respiratorio medido llegue a 0 Joule/L y los músculos respiratorios queden totalmente descargados. Brochard y colaboradores en 1989 reportaron que una presión de soporte alrededor de $15 \mathrm{cmH}_{2} \mathrm{O}$ desencadenaba una carga muscular óptima correlacionado con un trabajo respiratorio realizado por el paciente de 0,52+/-0,12 Joule/L, además encontró niveles de descarga parcial de los músculos respiratorios con $18 \mathrm{cmH}_{2} \mathrm{O}$ de presión de soporte y una descarga total muscular con $30 \mathrm{cmH}_{2} \mathrm{O}$ de presión de soporte.

Para individuos con respiración espontanea en condiciones normales el cambio en la presión muscular respiratoria es de aproximadamente $5 \mathrm{cmH}_{2} \mathrm{O}$, con lo cual se llevaría a asegurar que niveles por debajo de este nivel se considerarían subterapéuticos. Otros estudios más recientes aseguran que la presión de soporte ideal está alrededor de $12 \mathrm{cmH}_{2} \mathrm{O}$, dado que logra disminuir el esfuerzo respiratorio extra impuesto al paciente y de igual manera mejora la ventilación en procesos hipercápnicos. Al relacionar los datos 
recopilados en esta investigación con lo expuesto anteriormente se puede aseverar que la presión soporte utilizada al instaurar la VMNI, en estas dos UCIs de la cuidad de Bogotá, generó en sus usuarios un apoyo parcial $\left(10 \mathrm{cmH}_{2} \mathrm{O}\right)$, más hacia los niveles de postcarga diafragmática que hacia los de descarga, en ningún caso se usó para llevar a una descarga total donde se obtendría un trabajo respiratorio reducido a 0 .

Otra variable interesante en el uso de la VMNI es la sensibilidad inspiratorio o trigger, se relaciona con el esfuerzo respiratorio que genera el paciente para iniciar el ciclo respiratorio y guarda íntima concordancia con la actividad del centro respiratorio. Se expresa cómo la presión o flujo inspiratorio generada por el paciente con la vía aérea ocluida durante los primeros $100 \mathrm{~ms}$ del inicio de la inspiración y es una de las variables más importantes en el proceso de sincronía pacienteventilador (Mas \& Masip, 2014).

Para que la efectividad del trigger sea ideal influyen factores intrínsecos y extrínsecos, los primeros conciernen directamente a la velocidad y sensibilidad del transductor de presión que lleva a un esfuerzo inspiratorio inicial antes de obtener el disparo del mismo ventilador; los factores extrínsecos están asociados a aquellos que generan afectaciones de las resistencias interpuestas entre paciente y ventilador, siendo estos últimos los más importantes a la hora de valorar su efectividad en la interacción pacienteventilador sometido a VMNI.

Este parámetro de control ventilatorio en la VMNI se puede proporcionar al paciente de dos formas, y su valor base dependen únicamente de la casa fabricante del ventilador mecánico; se puede aportar por flujo o por presión. Algunos trabajos investigativos revisados favorecen los sistemas ciclados por flujo con respecto a los de presión para disminuir el trabajo respiratorio del paciente sometido a VMNI,

Para que el ventilador conceda el flujo de aire programado al paciente, este debe generar una presión o flujo mínimo $\left(0,5-1,0 \mathrm{cmH}_{2} \mathrm{O}\right.$ o $\left.0,5-1,0 \mathrm{~L} / \mathrm{s}\right)$, para que se dé la apertura de válvula inspiratorio, acrecentando así la sensibilidad del disparo, el cual incitará a la disminución del trabajo respiratorio. Si por alguna razón se aumentara dicha presión o flujo (2-5 $\mathrm{cmH}_{2} \mathrm{O}$ o 2-5 L/s), el paciente compensaría realizando mayor esfuerzo inspiratorio, con el consiguiente incremento del trabajo respiratorio y mayor desacople ventilatorio (Ferrero, 2008). La literatura no sugiere un valor puntual de recomendación, aunque las casas fabricantes de los ventiladores mecánicos aconsejan un valor de $2 \mathrm{cmH}_{2} \mathrm{O}$ o $2 \mathrm{~L} / \mathrm{s}$ como patrón de inicio.

En este estudio se encontró que en las dos UCIs se usó la sensibilidad ciclada por flujo únicamente, aspecto apoyado en la literatura mundial como recomendación primaria de uso frente al ciclado por presión, y tuvo un valor de $2 \mathrm{~L} / \mathrm{s}$, tal vez asociado a las recomendaciones de las casas fabricantes de los ventiladores mecánicos utilizados.

Algunas investigaciones recientes sugieren que el éxito en el uso de la VMNI está ligado al confort que el paciente presenta con la interfaz que se utiliza; la interfaz se define como un dispositivo que facilita la entrada del aire presurizado a través de la cara del paciente, debe ser cómoda, ligera, fácil de colocar y que no produzca dolor ni alergias, dado esto el mercado ofrece gran variedad entre mascaras nasales, oronasales y de total cobertura (Liesching, Kwok \& Hill, 2003).

Rodríguez y colaboradores en 2012 dedujeron que la máscara oronasal generaba mayor confort a los pacientes en comparación con la nasales y dicho factor favorecía la tolerancia al tratamiento interpuesto con la VMNI. Además aumentan la eficacia de la VMNI al minimizar las fugas y son más apropiadas en pacientes agudos. (Del Castillo, Galán, Gordillo \& Mateos, s/f). En este estudio se evidenció que en el $94 \%(n=29)$ de los pacientes utilizaron máscaras oronasales, con esto se deduce que en las dos UCIs analizadas se adoptan las recomendaciones con relación al uso de las interfaces de la VMNI.

Cabe resaltar que el sesgo en este estudio estuvo asociado con el desenlace de los pacientes que se sometieron al uso de la VMNI, para relacionar la evolución con el tratamiento llevado a cabo durante dicha terapéutica, aunque, durante el periodo de 
recolección de datos ninguno de los pacientes presentó complicaciones directamente relacionadas con la VMNI.

\section{CONCLUSIONES}

La VMNI es una medida terapéutica alternativa de uso frecuente al interior de las UCIs ofrecida a pacientes que por diferentes razones requieren un soporte ventilatorio, garantizando una baja incidencia de complicaciones al compararla con la ventilación mecánica invasiva.

Su uso se planteó hace varias décadas con un importante aguje y evidencia clínica a inicios de los 90, hoy en día los referentes internacionales que recomiendan su uso como una excelente alternativa aseguran que la adecuada selección de los pacientes, la toma de decisión de su inicio y el adecuado manejo y monitoria por parte del personal especializado son los pilares de este modo ventilatorio.

La VMNI se considera un tratamiento de primera línea con evidencia alta y grado de recomendación 1A en la falla respiratoria hipercápnica asociada a la presencia de EPOC y enfermedad neuromuscular, y en falla respiratoria hipoxémica asociada a edema pulmonar cardiogénico de origen valvular.

Finalmente la literatura mundial refiere que los modos ventilatorios de mayor uso al instaurar la VMNI son el BIPAP y CPAP, y en su uso de debe tener en cuenta el objetivo de su utilización y el tipo de falla o disfunción pulmonar que llevó a su aplicación; es decir, si la causa que llevó al uso de la VMNI fue una falla o disfunción hipercápnica, el objetivo principal será mejorar la ventilación alveolar, por tanto el modo de recomendación nivel I o alta es el BIPAP; por el contrario, si la causa que llevó al uso de la VMNI fue una falla o disfunción hipoxémica, el objetivo principal será mejorar la oxigenación, por tanto el modo de recomendación nivel I o alta es el CPAP.

\section{REFERENCIAS}

Artacho, R., Guzman, J., Lopez, S., Garcia, F., Caballero, F., Lopez, M., \& Del Campo, E. (2015). Ventilación no invasiva con presión de soporte con volumen asegurado en un paciente con enfermedad pulmonar obstructiva crónica (EPOC) agudizada. Revista de Patología Respiratoria, 18(1), 33-34.

Antonelli, M., Conti, G., Esquinas, A., Montini, L., Maggiore, S. M., Bello, G., Meduri, G. U. (2007). A multiple-center survey on the use in clinical practice of noninvasive ventilation as a first-line intervention for acute respiratory distress syndrome. Critical Care Medicine, 35(1), 18-25.

Brochard, L., Harf A. \& Lorino H. (1989). Inspiratory pressure suport prevents diaphragmatic fatigue during weaning from mechanical ventilation. American Review of Respiratory Disease, 139(1), 513.

Curtis, J. R., Cook, D. J., Sinuff, T., White, D. B., Hill, N., Keenan, S. P., Society of Critical Care Medicine Palliative Noninvasive Positive VentilationTask Force. (2007). Noninvasive positive pressure ventilation in critical and palliative care settings: understanding the goals of therapy. Critical Care Medicine, 35(3), 932-939.

Del Castillo Otero, D., Galán, C. C., Gordillo, M. A., \& Mateos, F. V. (s/f). Ventilación mecánica no invasiva. EB04-13 NEUMOSUR. Recuperado a partir de http:// neumosur.net/files/EB04-13\%20VMNI.pdf

Delclaux, C., L'Her, E., Alberti, C., Mancebo, J., Abroug, F., Conti, G., Brochard, L. (2000). Treatment of acute hypoxemic nonhypercapnic respiratory insufficiency with continuous positive airway pressure delivered by a face mask: A randomized controlled trial. JAMA, 284(18), 2352-2360.

Demoule, A., Girou, E., Richard, J.-C., Taillé, S., \& Brochard, L. (2006). Increased use of noninvasive ventilation in French intensive care units. Intensive Care Medicine, 32(11), 1747-1755.

Ferrer, M., Esquinas, A., León, M., González, G., Alarcón, A., \& Torres, A. (2003). Noninvasive ventilation in severe hypoxemic respiratory failure: a randomized clinical trial. American Journal of Respiratory and Critical Care Medicine, 168(12), 1438-1444.

Ferrero, G. (2008). XII. Modos ventilatorios en ventilación no invasiva. Revista chilena de enfermedades respiratorias, 24(3), 240-250.

Hannan, L. M., Dominelli, G. S., Chen, Y.-W., Darlene Reid, W., \& Road, J. (2014). Systematic review of non-invasive positive pressure ventilation for chronic respiratory failure. Respiratory Medicine, 108(2), 229-243.

Kacmarek R. (1988). The role of pressure support ventilation in reducing work of breathing. Respiratory Care. 88, 99-120. 
Keenan, S. P., Sinuff, T., Burns, K. E. A., Muscedere, J., Kutsogiannis, J., Mehta, S. Canadian Critical Care Trials Group/Canadian Critical Care Society Noninvasive Ventilation Guidelines Group. (2011). Clinical practice guidelines for the use of noninvasive positive-pressure ventilation and noninvasive continuous positive airway pressure in the acute care setting. CMAJ: Canadian Medical Association Journal = Journal de l'Association Medicale Canadienne, 183(3), E195-214.

Keenan, S. P., Sinuff, T., Cook, D. J., \& Hill, N. S. (2004). Does noninvasive positive pressure ventilation improve outcome in acute hypoxemic respiratory failure? A systematic review. Critical Care Medicine, 32(12), 2516-2523.

Liesching, T., Kwok, H., \& Hill, N. S. (2003). Acute applications of noninvasive positive pressure ventilation. Chest, 124(2), $699-713$

Mas, A., \& Masip, J. (2014). Noninvasive ventilation in acute respiratory failure. International Journal of Chronic Obstructive Pulmonary Disease, 9, 837-852.

Masclans, J. R., Pérez, M., Almirall, J., Lorente, L., Marqués, A., Socias, L., H1N1 GTEI/SEMICYUC Investigators. (2013). Early non-invasive ventilation treatment for severe influenza pneumonia. Clinical Microbiology and
Infection: The Official Publication of the European Society of Clinical Microbiology and Infectious Diseases, 19(3), 249-256.

Parola, D., Romani, S., Petroianni, A., Locorriere, L., \& Terzano, C. (2012). Treatment of acute exacerbations with non-invasive ventilation in chronic hypercapnic COPD patients with pulmonary hypertension. European Review For Medical And Pharmacological Sciences, 16(2), 183-191.

Rodríguez Álvarez, N. I., Muguercia Sarracén, K., Fabré de Armas, U., Esquivel Lussón, R., \& Zamora Cabezas, L. (2012). Ventilación mecánica no invasiva en la enfermedad pulmonar obstructiva crónica agudizada. (Spanish). Noninvasive mechanical ventilation in the severe chronic obstructive lung disease. (English), 16(12), 47-55.

Thille, A. W., Contou, D., Fragnoli, C., Córdoba-Izquierdo, A., Boissier, F., \& Brun-Buisson, C. (2013). Non-invasive ventilation for acute hypoxemic respiratory failure: intubation rate and risk factors. Critical Care (London, England), 17(6), R269-R269.

Truwit, J., \& Epstein, S. (Eds.). (2011). A practical Guide To Mechanical Ventilation. UK: John Wiley \& Sons, Ltd.

Uña, R., Ureta, P., Uña, S., Maseda, E., \& Criado, A. (2005). Ventilacion mecanica no invasiva. Res. Esp. Anestesiol. Reanim, 2, 88-100. 